\title{
Comparison of Magnetic Resonance Cholangiopancreatography (MRCP) with Ultrasonography (USG) and Computed Tomography (CT) in the Evaluation of Patients with Obstructive Jaundice
}

\begin{abstract}
Anand Rajamani ${ }^{1}$, Vijay Prabhu $\mathbf{R}^{2}$, Abubacker Sulaiman $\mathbf{F}^{3}$, Divya $\mathbf{Y}^{4}$, Lavanya $\mathbf{G}^{5}$, Saravanan $\mathbf{K}^{6}$
${ }^{1}$ Assistant Professor, Department of Radio Diagnosis, Chettinad Hospital and Research Institute, ${ }^{2}$ Assistant Professor, Department of Radio Diagnosis, Chettinad Hospital and Research Institute, ${ }^{3}$ Professor, Department of Radio Diagnosis, Chettinad Hospital and Research Institute, ${ }^{4}$ Final year Postgraduate, Department of Radio Diagnosis, Chettinad Hospital and Research Institute, ${ }^{5}$ Senior resident, Department of Radio Diagnosis, Chettinad Hospital and Research Institute, ${ }^{6}$ Senior resident, Department of Radio Diagnosis, Chettinad Hospital and Research Institute, India
\end{abstract}

Corresponding author: Dr. Vijay Prabhu R, Assistant Professor, Department of Radio Diagnosis, Chettinad Hospital and Research Institute, Kelambakkam, Chennai - 603103. India

DOI: http://dx.doi.org/10.21276/ijcmsr.2019.4.3.15

How to cite this article: Anand Rajamani, Vijay Prabhu R, Abubacker Sulaiman F, Divya Y, Lavanya G, Saravanan K. Comparison of magnetic resonance cholangiopancreatography (MRCP) with ultrasonography (USG) and computed tomography (CT) in the evaluation of patients with obstructive jaundice. International Journal of Contemporary Medicine Surgery and Radiology. 2019;4(3):C69-C73.

\section{A B S T R A C T}

Introduction: Obstructive jaundice is a widespread clinical problem. It has been documented as one of the primary cause of increased mortality and morbidity. Although clinical data such as history, physical examination, and laboratory tests can differentiate between intra hepatic and extra hepatic obstruction in $90 \%$ of patients, the cause and site of obstruction is diagnosed by imaging modalities. The frequently used imaging modalities include Ultrasonography (USG), Computed Tomography (CT), Endoscopic Retrograde Cholangiopancreatography (ERCP) and Magnetic Resonance Cholangiopancreatography (MRCP). Percutaneous Transhepatic Cholangiography (PTC) is used for drainage procedures. Study aim to appraise the diagnostic accuracy of MRCP in detecting the cause and level of obstruction in patients suffering from with Obstructive Jaundice.

Material and methods: A total no. of 25 patients suffering from obstructive jaundice of all age groups and either sex who were referred to Department of Radio-Diagnosis, Chettinad Hospitals and Research Institute were included in this study.

Results: Of the twenty five patients, ten patients had benign causes of obstructive jaundice while fifteen patients had malignant causes of obstructive jaundice. MRCP had an accuracy of $97 \%$ in detecting the cause of obstructive jaundice. In diagnosing the site of obstruction MRCP had an accuracy of $100 \%$.

Conclusion: In the diagnosis of obstructive jaundice and to know the cause, site and extent of the lesion MRCP being a non invasive, non ionizing procedure seems to be a better choice. The only drawback of MRCP is the cost involved and the availability. The limitation of the study is the small sample size and that ERCP correlation for these patients was not done.

Keywords: MRCP, Obstructive Jaundice, Cholangiocarcinoma

\section{INTRODUCTION}

Obstructive jaundice is one of the widespread clinical problems which has increased incidence of mortality and morbidity. Intra hepatic and extra hepatic obstruction in $90 \%$ of patients, can be made out through suitable clinical data but the cause and site of obstruction is diagnosed mostly by imaging modalities. The main aim of any imaging modality in obstructive jaundice is to identify the presence of obstruction, its location, extent, possible etiology.

The expanding spectrum of therapeutic options for the jaundiced patient has made it necessary for the radiologist to do more than simply discriminating between obstructive and non-obstructive jaundice. Correct choices among therapeutic options usually rest upon a precise assessment of etiology, location, level and extent of disease. ${ }^{1,2}$

The commonly used imaging modalities include Ultrasonography (USG), Computed Tomography (CT), Endoscopic Retrograde Cholangiopancreatography (ERCP) and Magnetic Resonance Cholangiopancreatography (MRCP). Percutaneous Transhepatic Cholangiography (PTC) is used for drainage procedures. ${ }^{3}$

Radiological imaging is vital in making a decision in management of patients with Obstructive Jaundice. Imaging choices available in diagnosing acute pancreatitis include USG, computed tomography (CT), Magnetic Resonance imaging (MRI), MRCP and ERCP. The choice depends 
upon the reason for investigation. ${ }^{4}$

Magnetic Resonance Cholangiopancreatography with its inherent high contrast resolution, rapidity, multiplanar capability and virtually artifact free display of anatomy and pathology, is proving to be imaging of choice in these patients. The quality of images obtained is comparable with those of direct cholangiography procedure like ERCP, which is considered as standard of reference in ductal pathologies. It has proved effective in demonstrating bile duct dilatation, stricture and Choledocholithiasis (Fig-1). The initial results with MR cholangiopancreatography studies were achieved with gradient echo sequences by using a steady-state free precession techniques. The latest imaging techniques for MRCP are Rapid Acquisition with relaxation Enhancement (RARE) and Half-Fourier Acquisition Single-Shot TurboSpin-Echo (HASTE). ${ }^{5,6}$

Though Ultrasonography and CT are non invasive, they have their drawbacks as well. USG is ineffective in accurately diagnosing the site of obstruction in most cases. CT has an increased risk of radiation and is also not sufficiently sensitive for detecting stones. ERCP and PTC are complicated procedures and require technical expertise and contrast media. Also several complications from the procedure may arise. $^{7}$

Study aimed to appraise the diagnostic accuracy of MRCP in detecting the cause and level of obstruction in patients suffering from with Obstructive Jaundice.

\section{MATERIAL AND METHODS}

A total of 25 patients with clinical history and symptoms of obstructive jaundice irrespective of age and sex who were referred to Department of Radio-Diagnosis, Chettinad Hospitals and Research Institute were included in our study. The inclusion criteria being the patients who were clinically diagnosed with obstructive jaundice.

\begin{tabular}{|l|c|c|}
\hline Sex & No of cases & Percentage (\%) \\
\hline Male & 11 & 44 \\
\hline Female & 14 & 56 \\
\hline Total & 25 & 100 \\
\hline
\end{tabular}

Table-1: Demonstrating Sex incidence in the studied population

\begin{tabular}{|l|c|c|}
\hline Type of lesion & No of cases & Percentage (\%) \\
\hline Benign & 10 & 40 \\
\hline Malignant & 15 & 60 \\
\hline Total & 25 & 100 \\
\hline Table-2: Benign versus Malignant causes of obstructive jaun- \\
dice in the studied population
\end{tabular}

Patients who were not suitable for MRI study due to claustrophobia, pregnancy or MR incompatibility were excluded from the study.

The age group of the patients varied from 21 to 86 . There was no obvious sex in relation to obstructive jaundice in our study. Out of twenty five patients, ten patients were diagnosed to have benign lesions while fifteen were diagnosed with malignant lesions. Among these fifteen patients with malignant lesions, MRCP had diagnosed all the fifteen. In diagnosis of the site of obstruction, MRCP was accurate in all twenty five patients.

The ethics committee of our institute approved this prospective study. Informed consent was taken from all patients undergoing this study. We prospectively studied 25 patients (14 females and 11 males) over a period of one year at Chettinad Hospitals and Research Institute. Initial USG evaluation was followed by CECT and MRI/MRCP, however in patients with Obstructive Jaundice with CBD calculi as diagnosed on USG,CT was performed if required. Transabdominal ultrasonography was done with convex 1 to $5 \mathrm{Mhz}$ probe on GE Voluson E8 followed by Contrast enhanced Computed Tomography (CECT) done on multislice CT with collimation of $2 \mathrm{~mm}$. However in cases of benign pathologies where USG findings were unequivocal CECT was not done to avoid unnecessary radiation exposure. MRCP was done in all patients on 3 Tesla MRI using our standard MRCP protocol.

\section{Inclusion Criteria}

Patients age between 21 to 86

Patients who have given Consent form

\section{Exclusion Criteria}

Patients less than 12 years of age.

With contraindications to MRI.

Patients with Prehepatic/Hepatic Jaundice.

\section{STATISTICAL ANALYSIS}

The Results obtained was expressed as percentages and variables as required. Microsoft office 2007 was used for the data analysis.

\section{RESULTS}

The table- 1 demonstrates that there was no obvious sex predilection among the patients affected with obstructive jaundice.

Table 2 shows that there was almost equal predilection for both benign and malignant causes of obstructive jaundice.

The illustration gives us a graphic representation of the common causes of obstructive jaundice. CBD calculi were the most common cause of obstructive jaundice followed by

\begin{tabular}{|l|c|c|c|c|c|}
\hline Modality & Sensitivity (\%) & Specificity (\%) & Positive Predictive Value (\%) & Negative Predictive Value (\%) & Accuracy (\%) \\
\hline MRCP & 94 & 100 & 100 & 94 & 97 \\
\hline \multicolumn{7}{|c|}{ Table-3: Diagnostic values of MRCP in benign causes of Obstructive Jaundice } \\
\hline
\end{tabular}

\begin{tabular}{|l|c|c|c|c|c|}
\hline Modality & Sensitivity (\%) & Specificity (\%) & Positive Predictive Value (\%) & Negative Predictive Value (\%) & Accuracy (\%) \\
\hline MRCP & 100 & 94 & 94 & 100 & 97 \\
\hline \multicolumn{7}{|c|}{ Table-4: Diagnostic values of MRCP in malignant causes of Obstructive Jaundice } \\
\hline
\end{tabular}




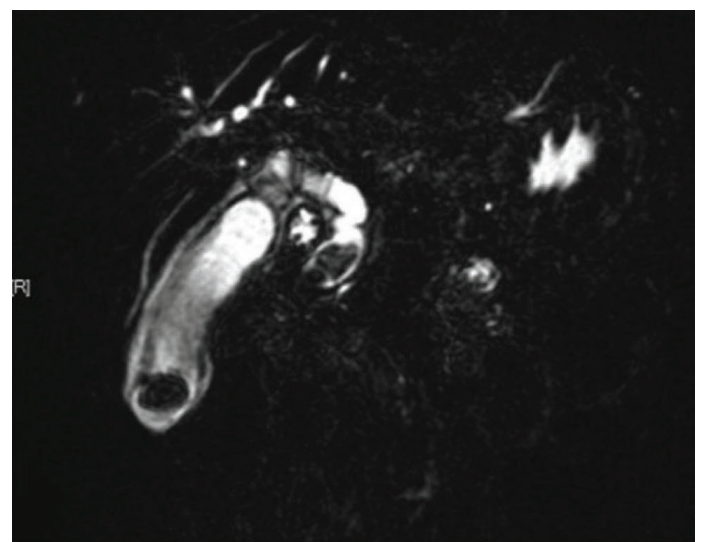

Figure-1: Case of Cholelithiasis with Choledocholithiasis

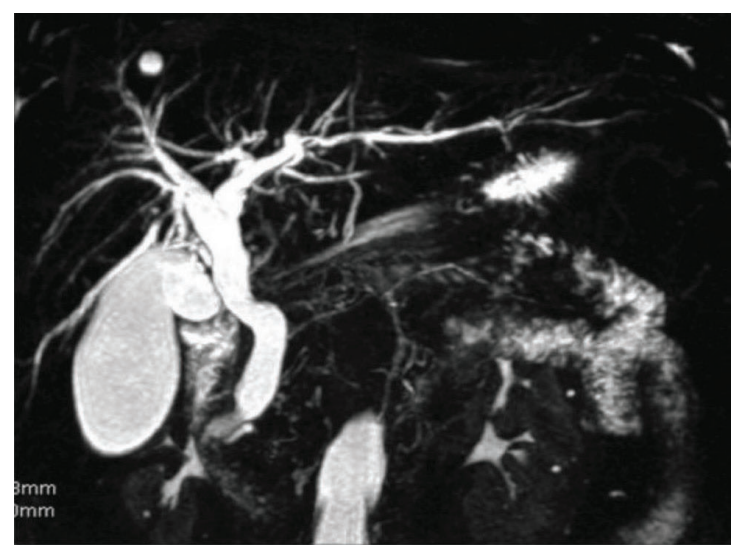

Figure-2: MRCP showing dilatation of the intramural portion of the distal common bile duct suggestive of Choledochocele
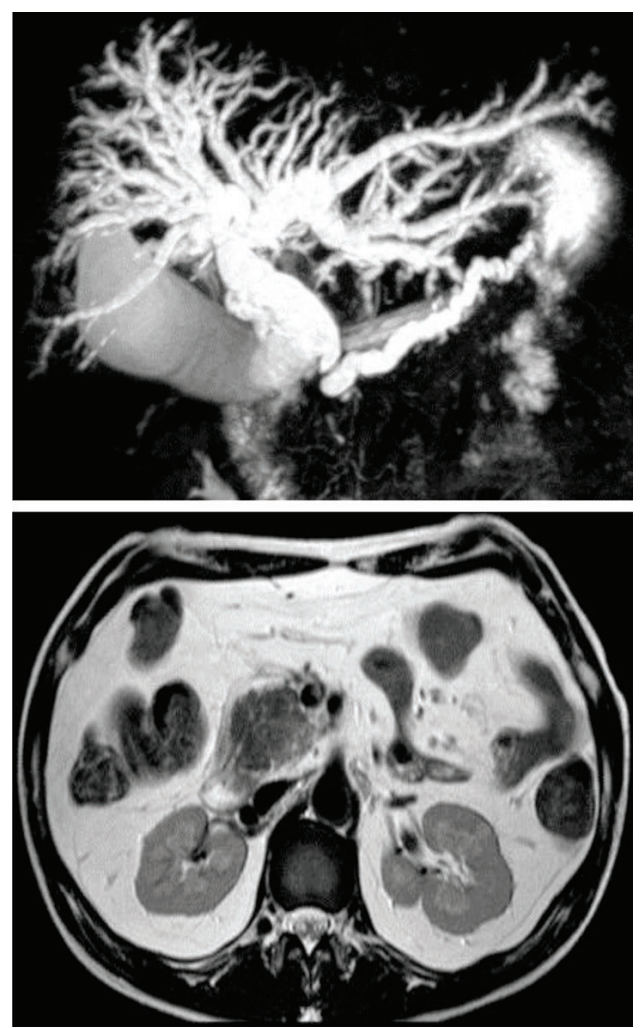

Figure-3a: Case of CA head of pancreas; Figure-3b: MRCP and T2W MRI showing dilatation of intra, extra hepatic and pancreatic duct with abrupt tapering at the Peri Ampullary region and hetero intense lesion in the head of pancreas.
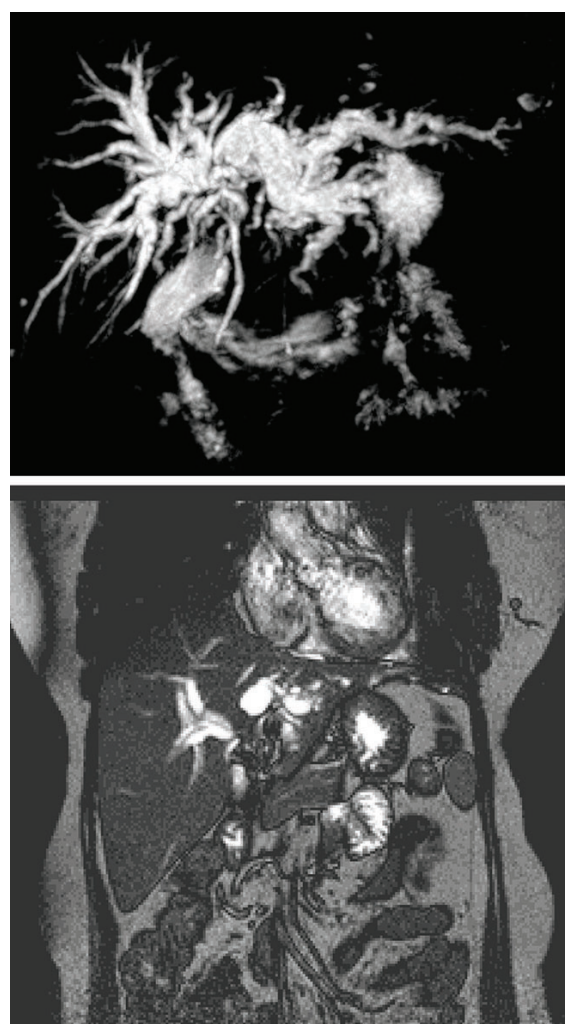

Figure-4a: Case of klatskin's tumour; Figure-4b: MRCP and T2W MRI showing dilatation of intra hepatic ducts with an ill defined tumor confined to confluence of the ducts

periampullary and $\mathrm{Ca}$ Head of Pancreas

From the table -3 it is inferred that MRCP has the highest accuracy for detecting benign lesions. The sensitivity of MRCP was $94 \%$ but the specificity was high as $100 \%$.

The specificity of MRCP was $100 \%$. Thus MRCP was a specific investigation for benign lesions of obstructive jaundice.

MRCP was again the most accurate investigation with an accuracy upto $97 \%$. The sensitivity and specificity of MRCP was high making it more accurate (table-4).

\section{DISCUSSION}

With the introduction of MRCP for diagnosing the biliary and pancreatic ductal pathologies, invasive procedure such as ERCP can be avoided. MRCP is used as a second line investigation following USG in patients with obstructive jaundice. ${ }^{8}$ MRI combined with MRCP is the safe and cost effective investigation in evaluating the patients with significant biliary duct dilatation or congenital biliary tree abnormalities. ${ }^{9}$ Single shot MRCP was highly sensitive (70 to $80 \%$ ) and specific in detecting lesions in bile duct and pancreatic duct. ${ }^{10}$

Most presenting complaints were jaundice, abdominal pain, passing pale colored stools and itching. In our study 25 patients with clinical history of obstructive jaundice were studied. On clinical examination Icterus was the most common sign elicited. The youngest patient in our study was 27 years old female who was suffering from Carcinoma of pancreatic head with PD dilatation. The oldest patient was seventy five years old and was suffering from periampullary carcinoma. The average age of patients with benign lesions 
and malignant lesions was in the fifth decade.

Six patients out of 25 were diagnosed with CBD and GB calculi which were accurately diagnosed by MRCP. Our study is in concordance with Soto et al 2000 study, they studied that detecting biliary calculi through MRCP is $94 \%$ sensitive and $100 \%$ specific. ${ }^{11}$ Stephan et al did a study and found that diagnosing CBD calculus was $87 \%$ sensitive.

Three patients were diagnosed with Stricture disease. MRCP was $100 \%$ accurate in diagnosing the benign nature of the stricture in all 3 cases. The length of the stricture segment was well appreciated and helps in differentiating it from malignant stricture. MRCP to be extremely accurate in showing pancreatic dilatation, strictures, stones, cystic dilatation with sensitivity approaching $100 \% .^{12}$ Our study is in concordance with Bhatt et al study they evaluated that $\mathrm{MRCP}$ is $100 \%$ accurate in diagnosing benign $\mathrm{CBD}$ stricture. ${ }^{14}$

One case of anatomical variant, a case of Choledochocele cyst was present in our study (fig-2). It was diagnosed accurately by MRCP. Our study is in concordance with Bhatt et al study who found $100 \%$ accuracy in diagnosing anatomical variants through MRCP.

Among the malignant lesions there were 2 cases of Head of Pancreas (Fig 3a) tumor and 4 cases of Periampullary Ca. MRCP accurately diagnosed all the six cases. Though MRCP alone could not clinch the diagnosis a few sequences of MRI was required to diagnose accurately the malignant lesions. Our study is in accordance with Anderrson et al 2005 study, they found MRCP is $90 \%$ accurate for diagnosing periampullary growth. ${ }^{14} \mathrm{MRCP}$ and T2W MRI showed dilatation of intra, extra hepatic and pancreatic duct with abrupt tapering at the Peri Ampullary region and hetero intense lesion in the head of pancreas (fig: 3b).

In 2 patients with extra hepatic Cholangiocarcinoma, MRCP with the help of conventional MRI is best at diagnosing all the cases with 100\% accuracy. MRCP using breath-hold SSFSE sequence is exact in identifying the level of obstruction and the underlying tumor. ${ }^{15}$

Klatskin's tumor was found in three patients (Figure-4a), and MRCP showed 100\% accuracy as studied by Bhatt et al 2005 in diagnosing klatskin's tumor which is $100 \%$ accurate by MRCP. ${ }^{13} 3$ patients with $\mathrm{Ca}$ of Gallbladder were diagnosed accurately by MRCP.

In patients with obstructive jaundice, ERCP is considered as the standard of reference in imaging, since it provides high resolution of biliary tree and pancreatic duct. The main advantage of ERCP is that it can perform therapeutic interventional procedures such as removal of stone, stricture dilatation, and stent placement. Highly skilled and experienced endoscopist is essential to perform this procedure. Visualization of biliary tree proximal to severe obstruction may not be possible almost always. Post procedural morbidity and mortality is significant. Procedure may not be performed in critically ill patients. ${ }^{7} \mathrm{MRCP}$ can clearly provide valuable information when ERCP is unsuccessful or inaccurate. ${ }^{16}$

Study conducted by Al-Obaidi et al., ${ }^{17}$ showed higher sensitivity (100\%), specificity (98.5\%), accuracy (98.7\%) of MRI/MRCP for cases with benign stricture as compared to sensitivity of USG (44.4\%) which is consistent with present study.

Andersson $\mathrm{M}$ et al., ${ }^{14}$ concluded in their study that MRI with MRCP was more accurate than $\mathrm{CT}$ in differentiating between malignant and benign lesions in patients with suspected periampullary tumors. This is consistent with present study where MRI/MRCP showed $100 \%$ accuracy in diagnosing cases with periampullary carcinoma.

MRCP is the only choice of investigation in imaging patients suffering from obstructive jaundice, as Helical CT and USG has few limitations invasiveness and complications. With addition of conventional MRI, MRCP becomes still more superior. Dynamic MR imaging is superior to helical CT in the preoperative detection and evaluation of local extension of tumor. ${ }^{18}$

Though MRCP is evolving as the gold standard in biliary imaging there are several pitfalls in MRCP imaging. Familiarity with the findings and imaging techniques which lead to misdiagnosis may help prevent misinterpretation of MRCP images. Also ERCP correlation, if available would have given a complete analysis of all the diagnostic modalities of obstructive jaundice. ${ }^{19-23}$

\section{CONCLUSION}

$\mathrm{MRCP}$ is the non invasive, non ionizing procedure in diagnosing the cause for obstructive jaundice and detect the cause, site and extent of the lesion. Cost and the availability are the only drawbacks of MRCP. The limitation of the study is the small sample size and that ERCP correlation for these patients was not done. With the introduction of MRI guided interventions MRCP can become both diagnostic and therapeutic application in detecting and treating obstructing jaundice and biliary tract in the near future.

\section{REFERENCES}

1. Honickman SP, Mueller PR, Witternberg J, Simeone JF, Ferrucci JT, Cronan JJ, Van Sonnenberg E. Ultrasound in obstructive jaundice: prospective evaluation of site and cause. Radiology. May 1983;147(1):811-15.

2. Suraj Sonawane, Sachin Bagale, Sunil Patil. MRCP a problem solving diagnostic tool in pancreaticobiliary pathologies. International Journal of Contemporary Medicine Surgery and Radiology. 2018;3(2):144-148.

3. Buscail L, Escorrou J, Moreau J, Delvaux M, Louvel D, Lapeyre F, Tregant P, Frexinos J.Endoscopic ultrasonography in chronic pancreatitis: a comparative prospective study with conventional ultrasonography, computed tomography and ERCP. Pancreas.1995. 10(1):251-257.

4. Sharma V, Rana SS, Sharma RK, Kang M, Gupta $\mathrm{R}$ and Bhasin DK. A study of radiological scoring system evaluating extrapancreatic inflammation with conventional radiological and clinical scores in predicting outcomes in acute pancreatitis. Ann Gastroenterol 2015;28(3): 399-404.

5. Shadan A, Malik GM, Kamill MMA, Umar K, Showkat $\mathrm{H}$, Willayat A, et al. Role of MRCP in the evaluation of suspected biliary and pancreatic disease. JK Pract. 2011;16(1-2):19-23. 
6. Mary Kurian J, Kumar John P, Hegde P, Murthy C, Kumar A. A Comparative Evaluation of USG and MRCP Findings in Biliary and Pancreatic Pathologies. Int J Contemp Med Res 2017;4(1):212-215.

7. Caroline Reinhold and Patrice M. Bret: Current status of MRCP. AJR 1996; 166(3): 1285-1295.

8. G.J. Robinson et al: MRCP in obstructive jaundice. Radiology 1997, Imaging, Science \& Oncology, 124.

9. HO JT, Yap CK: Value of using HASTE sequence, Ann Acad. Med Singapore. May 1999; 28(3): 366-70.

10. K. Liberpoulos et al: The role of MRCP in the study of biliary diseases, Radiology 1997. Imaging, Science \& Oncology, 22.

11. Soto et al: comparision of three dimensional fast spin echo and single and multisection half fourier rapid acquisition with relaxation enhancement sequences. Radiology 2000;215(6):737-745.

12. Schwartz et al: Neoplastic Pancreato-biliary Duct obstruction: Evaluation with breath hold MRCP. AJR 1998; 170(1): 1491-1495.

13. Bhatt C, Shah P.S, Prajapati H.J, et al: Comparison of Diagnostic Accuracy between USG and MRCP in Biliary and Pancreatic Pathology. Ind J Radiol Imag 2005; 5:2:177-181.

14. Andersson M, Kostic S, Johansson M, Lundell L, Asztely M, et al: MRI combined with MR cholangiopancreatography versus helical CT in the evaluation of patients with suspected periampullary tumors: a prospective comparative study. Acta Radiologica 2005; 46(4): 16-27.

15. J.C. Varghese et al: The Diagnostic Accuracy of Magnetic Resonance Cholangiopancreatography and Ultrasound compared with direct Cholangiography in the detection of Choledocholithiasis. Clinical Radiology 1999; 54(1):604-614.

16. Regan et al: Choledocholithiasis: Evaluation with MRCP. AJR 1996; 167 (1):1441-1445.

17. Tomoaki Ichikawa, Hiroki Harodome, et al: Pancreatic Ductal Adenocarcinoma: Preoperative Assessment with Helical CT versus Dynamic MR imaging. Radiology1997; 202(3): 655-662.

18. Safa Al-Obaidi, Mohammed RidhaAlwan, Al-Hilli, Atheer, Adnan Fadhel. The Role of Ultrasound and Magnetic Resonance Imaging in the Diagnosis of Obstructive Jaundice. The Iraqi Postgraduate Medical Journal. 2007;6(1):7-17.

19. Hiroyuki Irie, Hiroshi Honda, Toshiro Kuroiwa, Kengo Yoshimitsu, Hitoshi Aibe, Kenji Shinozaki, Kouji Masuda. Pitfalls in MR Cholangiopancreatographic Interpretation. Radiographics 2001;21(2):23-37.

20. Marimuthu Veerasamy, A. Muthuvinayagam, K. Sugumaran. A study on extra hepatic biliary calculi. International Journal of Contemporary Medical Research 2017;4 (1):133-135.

21. Abhinandan Goswami, Mituban Gogoi, Mustafa Abdur Rahman. A study on biliary leakage after cholecystectomy. International Journal of Contemporary Medical Research 2017;4(5):1212-1215.

22. Chanda Bhaskara Rao, G. Hasanthi, J.S. Kishore, Srilekha, Bhavana Chanda. Paediatric choledochal cysts- management of thirty seven patients in paediatric surgery department of Guntur medical college. International Journal of Contemporary Medical Research 2017;4(12):1-5.

23. Ritesh Kondeti, Prashanth Thimme Gowda. A study on comparitive role of ultrasonogram and computed tomography in diagnosis of acute pancreatitis. International Journal of Contemporary Medicine Surgery and Radiology. 2018;3(3):C164-C167.

Source of Support: Nil; Conflict of Interest: None

Submitted: 12-06-2019; Accepted: 27-03-2019; Published online: 06-08-2019 